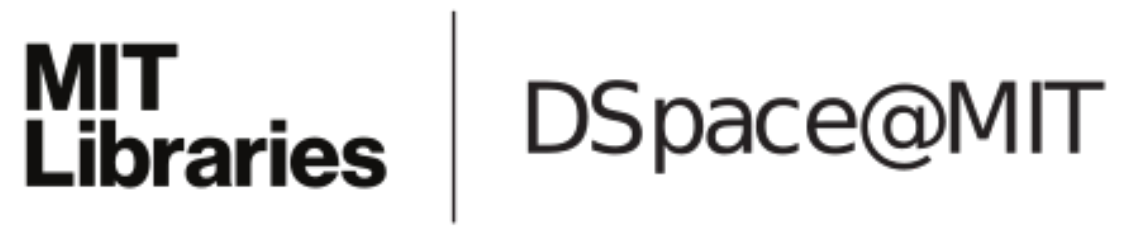

\author{
MIT Open Access Articles
}

\section{ProtoSpray: Combining 3D Printing and Spraying to Create Interactive Displays with Arbitrary Shapes}

The MIT Faculty has made this article openly available. Please share how this access benefits you. Your story matters.

Citation: Hanton, Ollie, Wessely, Michael, Mueller, Stefanie, Fraser, Mike and Roudaut, Anne. 2020. "ProtoSpray: Combining 3D Printing and Spraying to Create Interactive Displays with Arbitrary Shapes." Proceedings of the $2020 \mathrm{CHI}$ Conference on Human Factors in Computing Systems.

As Published: 10.1145/3313831.3376543

Publisher: ACM

Persistent URL: https://hdl.handle.net/1721.1/137376

Version: Author's final manuscript: final author's manuscript post peer review, without publisher's formatting or copy editing

Terms of use: Creative Commons Attribution-Noncommercial-Share Alike 


\title{
ProtoSpray: Combining 3D Printing and Spraying to Create Interactive Displays with Arbitrary Shapes
}

\author{
Ollie Hanton ${ }^{1}$, Michael Wessely ${ }^{2}$, Stefanie Mueller ${ }^{2}$, Mike Fraser $^{3}$, Anne Roudaut ${ }^{1}$
}

${ }^{1}$ University of Bristol, UK

\{oh12660, Anne.Roudaut\}

@bristol.ac.uk

\author{
${ }^{2}$ MIT CSAIL, Cambridge, MA, USA \\ \{wessely, stefanie.mueller\}
} @mit.edu

\author{
${ }^{3}$ University of Bath, UK \\ mcf35@bath.ac.uk
}
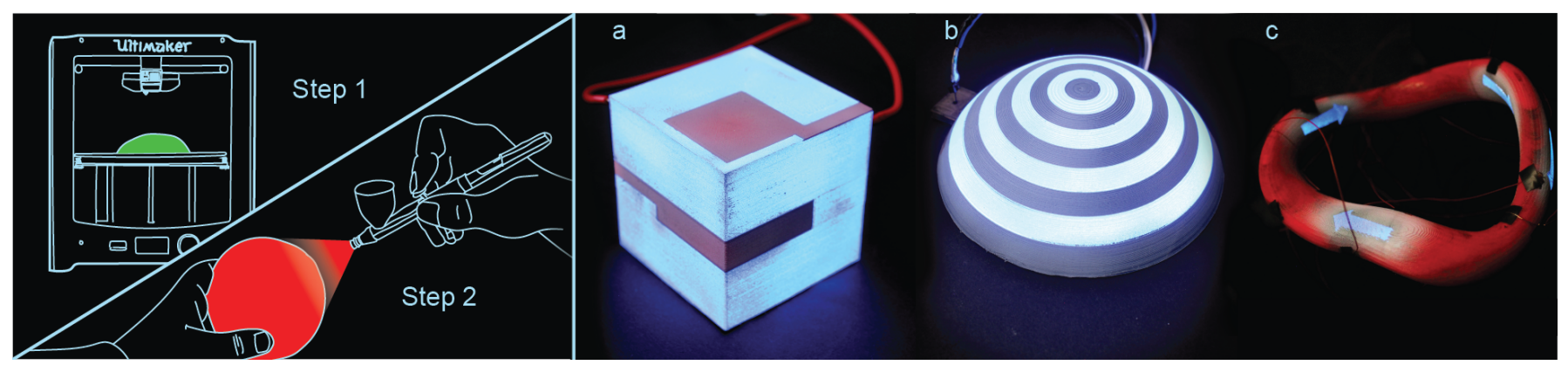

Figure 1. ProtoSpray is the first fabrication technique that combines 3D printing with spray coating to create interactive displays of arbitrary shapes. It uses mixed material printing of electrodes to create solid objects which are sprayed with layers of electroluminescent ink. Our prototypes (three of them shown (a-c)) demonstrate how ProtoSpray enables the creation of displays with complex curvatures, going further than any work done before.

\begin{abstract}
ProtoSpray is a fabrication method that combines 3D printing and spray coating, to create interactive displays of arbitrary shapes. Our approach makes novel use of 3D printed conductive channels to create base electrodes on 3D shapes. This is then combined with spraying active materials to produce illumination. We demonstrate the feasibility and benefits of this combined approach in 6 evaluations exploring different shaped topologies. We analyze factors such as spray orientations, surface topologies and printer resolutions, to discuss how spray nozzles can be integrated into traditional 3D printers. We present a series of ProtoSprayed objects demonstrating how our technique goes beyond existing fabrication techniques by allowing creation of displays on objects with curvatures as complex as a Mobius strip. Our work provides a platform to empower makers to use displays as a fabrication material.
\end{abstract}

\section{Author Keywords}

3D Printing, Spraying, Display, Electroluminescence, Rapid Prototyping, Fabrication

\section{CCS Concepts}

-Human-centered computing $\rightarrow$ Touch screens;

\section{INTRODUCTION}

3D printers have revolutionised the way we create interactive objects, allowing non-experts to prototype industrial quality

Permission to make digital or hard copies of all or part of this work for personal or classroom use is granted without fee provided that copies are not made or distributed for profit or commercial advantage and that copies bear this notice and the full citation on the first page. Copyrights for components of this work owned by others than ACM must be honored. Abstracting with credit is permitted. To copy otherwise, or republish, to post on servers or to redistribute to lists, requires prior specific permission and/or a fee. Request permissions from permissions@ acm.org.

CHI '20, April 25-30, 2020, Honolulu, HI, USA.

2020 Association of Computing Machinery.

ACM ISBN 978-1-4503-6708-0/20/04 ...\$15.00.

http://dx.doi.org/10.1145/3313831.3376543 products at home. Beyond conventional plastic printing, it is even possible to print functional objects such as capacitive touch sensors using conductive filament [43], speakers via printed piezoelectric material [20], objects conveying light via embedded optical fibers $[9,53]$ and actuators printed in dielectric elastomer [16].

Despite the abundance of new techniques, 3D printed displays are largely unexplored. Existing machines for printed electronics are confined to specialized labs as they rely on complex mechanisms beyond the scientific and financial reach of typical fabrication spaces and end users, such as nano-scale printing techniques [6] or Aerosol Jet approaches [44]. Consequently it is limiting for the design community to experiment with new forms of displays as they must rely on using projection $[2,7$, 40], or off-the-shelf display tessellations [4, 27].

Electroluminescent (EL) ink has gained popularity among the $\mathrm{HCI}$ community as a way to address this issue and explore new approaches to display fabrication. Ink can be deposited using methods such as screen printing [34, 50, 52], spin-coating [3] or bar coating techniques [35]. However, these processes are limited to substrates with flat topologies and can thus produce a limited range of display shapes. Hydro-printing [15] has been demonstrated to have the potential to create displays on 3D objects but this has not been successfully shown beyond a single EL cell on a gently curved surface. The topologies of shapes that can be created is thus still limited.

However, creating complex topologies with EL material is challenging. The material cannot be deposited directly using domestic 3D printers because: (1) EL requires uniform deposition to avoid unpredictable electrical behaviour and short circuits [3]; (2) deposition needs to be in thinner layers, for energy efficiency, than commodity $3 \mathrm{D}$ printer resolution allows 
[41]; (3) conductive electrodes must be made from optically transparent materials to allow light through from the EL layer, but 3D printers can't currently process these materials; and (4) issues arising from mixing with filament or high temperature extrusion risk loss of electrical properties [23]. This explains why 3D display fabrication has instead focused on non-electronically controlled materials such as thermochromic $[31,36,54]$ or photochromic inks $[21,39]$.

To address this we present ProtoSpray, a method to create lowfidelity displays with complex topologies using EL material. It combines $3 \mathrm{D}$ printing techniques with spray-coating to print electronic material without limiting the variety of topologies possible. It uses interconnected electrodes through 3D printing of conductive filament, supplying electrical power to displays. This paper focuses on output capabilities, but these displays do also support touch sensing. Spray coating allows the precise, thin and cohesive application required to create thin displays on irregular surfaces. By using mixed material printing our technique enables the creation of a variety of shapes, going beyond what other techniques have enabled so far.

Our work analyses the feasibility of combining mixed-media 3D printing and spray coating as a single fabrication technique and the extent to which it works with various form factors. We explore this with a total of six evaluations, starting by assessing feasibility, by using simple electrodes on flat surfaces. We then move onto non-planar surfaces, before creating more complex electrode patterns. We conclude with a range of ProtoSpray prototypes which demonstrate that our techniques can create a variety of shapes with complex curvatures.

We show for the first time that it is possible to fabricate touchsensitive displays embedded in 3D printed objects of arbitrary shapes. We believe our work is particularly relevant for the large community of HCI researchers and designers interested in pushing the vision of non-rectangular displays (e.g., tangible user interfaces, organic user interfaces, shape changing interfaces, data physicalisation) and we think it will address the increasing demand for free form displays that these communities require. We also hope to inspire other researchers into rethinking the way we fabricate displays and consider displays as a material, to the same extent as plastic or paint.

To summarise, our contribution is a new fabrication technique exploring (1) spraying active materials to create displays which has never been used in $\mathrm{HCI}$ before; and (2) using 3D printed conductive channelling as base electrodes and to display shape for EL cells, which has also not been carried out in any literature to our knowledge. We also contribute (3) six evaluations to investigate the feasibility of the approach and (4) a series of prototypes to demonstrate it.

\section{BACKGROUND}

We focus on accessible display fabrication before exploring in detail the cases of spray coating.

\section{D Printing Displays}

There are many 3D printers printing more than just thermoplastics. E.g. xPrint [49] focuses on printing materials that come in a liquid solution and solidify under chemical reaction or physical transformation, but is not focused on creating the thin films necessary for displays. Kong et al. present a
Quantum Dot LED array using additive manufacturing techniques, printed on a modified industrial robotic dispenser [25]. They focus on production of QD-LEDs of a competitive size and produce an array of these as a proof of concept, although not through an accessible fabrication approach. 3D printing projects which fabricate displays are scarce, but these include Colormod [39] which uses photochromic plastic filaments to create objects changing colour when an external light source shines on them. Auzinger et al. [6] propose 3D printing of nano-scale structures to create structural color, although such techniques require precise and inaccessible equipment.

\section{Rapid Prototyping of Displays}

According to Klamka et al. [24], display technologies are classified as pixel-addressable high-resolution displays (e.g. OLED or E-ink) or as segment displays consisting of predefined shapes that illuminate independently. Sweeney et al. [47] combine an E-ink display with a layer of photodiodes to explore the idea of displays as a material. Thermochromic paint is an accessible technology to create segment displays [36], although the color change is not immediately responsive.

Another accessible technology is electroluminescent (EL) material [30]. EL as a material for fabricating thin-film displays has been explored by Olberding et al. [34], using screen printing and inkjet techniques. Screen printing is a technique that entails spreading ink onto a substrate by wiping the ink over it (or a stencil). This method is also increasingly used within the HCI community to create printed electronics [26]. However, its limitation is that it can only fabricate flat displays, which limits the topologies that can be created to only bendable or rollable shapes (such as a cylinder) [33]. Similarly, EL cells are used in Skinmarks [51] on irregular surfaces but are created on a flat topology via screen printing, before application via transfer paper and only conformal to gently curved surfaces.

Low resolution alternatives exist, such as Graffiti Fur [46], which uses shading properties of fur change as the fibers are raised or flattened to render images. Sweepscreen [32] similarly uses magnetophoretic surfaces and a device with a row of electromagnets. Lastly, Lindlbauer et al. [28] create appearance changing devices by laser cutting sheets of polymerdispersed liquid crystal (PDLC) switchable diffuser.

\section{Hydro-Printing}

Hydro-printing is the current technique most closely related to ProtoSpray, as it can fabricate non-planar displays [15]. However, hydro-printing suffers from limitations that spraying does not, such as an inability to cover sharp edges, deep concavities or complex curvature as the authors report. Users must align the printed object to the liquid basin and stretch a 2D shape over a 3D frame [55], which can cause distortion on non-flat objects. Hydro-printing also precludes the annotation of 3D objects printed with soluble PVA support material, as the process dissolves the supports. Finally, ProtoSpray creates base electrodes within a substrate object (3D channelling) and then adds EL materials to their surface, rather than placing all materials on a pre-existing object. As a result, a greater range of potential designs can be produced with ProtoSpray as we need not account for base electrodes crossing each other. 


\section{Spraying Displays}

Spray coating is a known technique to rapidly prototype thinfilm displays, but this has never been applied to building interactive objects. Ink must be atomised into an aerosol and directed over a substrate. Spray-guns use compressed air to create a high velocity airflow through a static liquid. Air pressure, nozzle size, distance, motion and material viscosity are important parameters in creating a thin yet uniform layer. Research exists on fabrication of thin-film surfaces via spraying, that respond to stimuli [3, 10, 12, 14], although these approaches use flat substrates. Photo-Chromeleon explores spraying of photochromic inks to produce objects that can be re-programmed, over an extended time period, to display different patterns [21]. Spraying has strengths due its accessibility and ability to provide consistent thicknesses and uniform layers to irregular surfaces. Work has been done on automating parts of handheld spraying to improve the nonexpert production of sprayed images [11, 29, 45]. Combining 3D printing and spraying has been explored by Falco et al. [13] who imagined an FDM and inkjet machine for creating objects with conductive traces. We build on this concept, integrating the conductive trace stage into the $3 \mathrm{D}$ printing and using a airbrush spraying process to layer on displays. Spraying can also create thin films over non-planar surfaces. Sandström et al. [41] demonstrate spraying EL layers to create light sources on irregular surfaces using sprayed metal electrodes with no account of substrate or configurability of display.

\section{PROTOSPRAY CONCEPTS}

ProtoSpray involves 3D printing an object made of two materials (conductive and insulating) and then layering the components of an EL display using spray coating onto the object (Figure 2). We discuss the principles and advantages of our approach and provide details about the materials used and the fabrication.
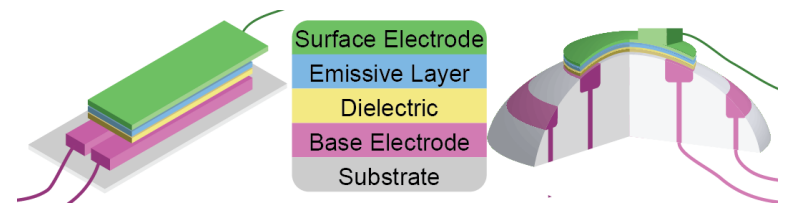

Figure 2. Traditional EL layering (left) compared to ProtoSpray layering, applied to an object without base electode masking (right). In traditional EL display creation, the base electrode is applied to the substrate as an ink. In ProtoSpray, without masking, the base object is 3D printed on a multimaterial printer with the base electrode embedded into the substrate. The other three layers are then sprayed onto the surface.

\section{ProtoSpray principle}

For an EL display to emit light it requires four layers: a conductive bottom electrode, an insulating dielectric layer, an insulating light-emitting layer, and a conductive top electrode. The bottom electrode is often made from a highly conductive metal, such as copper or silver ink. The dielectric layer is an electrical insulator, and must spread beyond the electrode layers to prevent short circuits between the top and bottom of the structure. The light-emitting layer is EL phosphor suspended in solvent. The top conductive layer must be transparent as with some polymers or metal oxides. To light up, an alternating current of around $200 \mathrm{~V}$ operates between the electrodes, across the dielectric layer, energizing the light-emitting layer.
A cell is an illuminated area of the display and an electrode can refer to either of the conductive layers (top/surface or bottom/base), connected to the power source. If a portion of any of the four layers is missing within a cell, it will not light up. If the conductive materials from separate electrodes come into contact with each other they will create a short circuit and none of the cell will function. It is thus important for the dielectric layer to be thin enough to allow the bottom layer below to energize the light-emitting layer above, but uniform enough to not allow short circuits between the layers [18].

\section{ProtoSpray eliminates masking}

ProtoSpray is based on the removal of masking which is a key technique for creating custom and distinct EL cells.

Traditional masking: Masks are commonly created via a physical stencil, tape or masking fluid between a material and the substrate. Masking performs two functions: the mask (1) gives a precise shape to the bottom electrode layer, which is used to shape the illuminating cell (though this can also be done by masking the light-emitting layer and/or top electrode); (2) prevents electrical interactions between layers by bounding electrical channels to and from cells. EL displays typically use a mask which shapes the bottom electrode connection sideways out of the display area along the substrate. This connection is subsequently masked while the dielectric, lightemitting and top electrode layers are sprayed, avoiding short circuits around the dielectric layer and allowing later electrode connection to the power supply. So, accurate masking is necessary to give the EL cell a particular visual effect, to prevent short circuits, and to route electrode connections appropriately. A similar end result to masking could be achieved by directing the atomised material in the spray plume, but this is hard to accomplish accurately [37]. There are two types of masking:

- Contact masking places the stencil physically against the substrate, creating a raised peak of sprayed material along the edge of the mask as droplets settle against the stencil's edge. For the base electrode of an EL display, this peak needs to be flattened by gentle sanding to avoid penetration through subsequent layers. Sanding the contact mask peak is a challenging process because too little will result in a short circuit through the dielectric layer, while too much can lead to inconsistent luminescence due to reduced material uniformity in the bottom electrode layer.

- Shadow masking [1] raises the stencil above the sprayed surface by a small amount, avoiding a peak on the edge of the sprayed material and instead creating a tapered edge. However, this technique reduces the spatial precision of the masking which increases the risk of a short circuit to another overlapping layer. Shadow masking also requires more space between cells because the edge doesn't anneal completely and so part of the sprayed area is too disconnected to act as an electrode. These costs are exacerbated by hand-spraying because manual movement of an airbrush produces more variance in angle than a mechanised approach, leading to less spatial precision between the stencil shape and the shape of the EL cell.

Eliminating masking: ProtoSpray eliminates masking by 3D printing base electrodes (channels) of conductive PLA, housed within the substrate object and printed simultaneously in a 
desired shape. By doing so we benefit from the printer resolution to reach similar or better precision than existing masking techniques, without requiring additional masking or sanding of the 3D printed shape. Masking by hand is a time intensive and skill-dependent process. Manual masking is not straightforward or easily replicable for objects which are strongly curved. It is also limited in scope with regards to being automated as a process and suffers from issues with scalability. For small cells, masking by hand is the easiest as there is no requirement for preparation of the surface. For larger non-flat cells, shadow masking is easier than contact masking, due to the challenge of making a stencil which is conformal to the surface of the base object, as well as having proportionally less error as size increases. Neither of the existing techniques are precise, replicable and suitable for 3D shapes.

\section{ProtoSpray's channeling benefits}

Figure 3 illustrates two ProtoSpray base electrode channels. Channeling conductive pathways in an object leaves the surface free of unwanted electrode areas and reduces the need for additional masking to avoid short circuits. Each channel has different parameters: channel width, channel length, channel orientation with respect to 3D printed layers, surface resolution (i.e. minimum size of channel ends), and channel tolerance (i.e. distance that channels must be apart to remain electrically distinct). Channeling builds upon work such as [42], proposing to permeate a 3D printed object with tubes for touch sensing. Here we use it for display purposes as well. We use conductive channels to direct current through an object, and $3 \mathrm{D}$ printed channel ends as connection points and final display shapes.

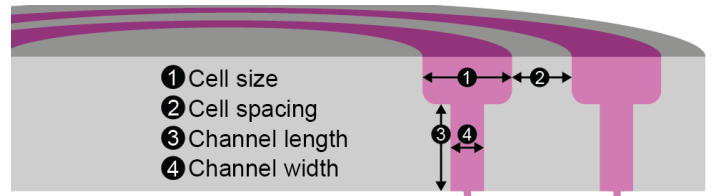

Figure 3. Channelled base electrodes in a 3D printed object.

Using conductive channels rather than 'on surface' electrodes has a number of advantages to object design. Using 3D printed channels allows digitisation of the process for defining cell shape, increasing the potential for fully automating the process. Routing a conductive pathway inside the object allows the only points on the surface to be the EL cells and the electrode attachment points, without the need for an on-surface conductive trace between the two. As a result, base electrode channels can cross each other in ways they wouldn't be able to in 2D. Advantages of limited conductive traces on an object's surface are: (1) a wider range of possible cell placement. This both opens design options and gives a potential for higher resolution of display due to denser cell placement, since space on the surface is no longer required for base electrodes merely for the attachment sites; (2) easier electrode attachment points can be 3D printed in a wider range of potential locations, being less dependent on segment sizes/shape/location; (3) improved spraying, as using the back of the object for channel attachment means less use of masking in spraying and so reducing the risk of a short circuit; and (4) reduced error as there is less base electrode area that could create contact with top electrode material which would lead to shorts, or with other base electrodes leading to cross-talk between cells. These benefits increase the range of designs and potential for ProtoSpray as a fabrication method, compared to what would otherwise be possible via spraying EL materials on pre-existing objects.

\section{ProtoSpray is an accessible fabrication tool}

Simplification of the spraying process by automation of printed base electrodes through 3D printed conductive channels, means that the EL fabrication approach is significantly less sensitive to user experience level and opens potential for a combined fabrication process.

Printing phase: the shape of the object is first printed in standard plastic, while light-emitting areas are deep-set $(\geq 1 \mathrm{~mm}$, depending on design shape) and printed in conductive plastic. Both phases of prints are carried out on mixed-media commercially available $3 \mathrm{D}$ printers. For each display cell on the surface, a conductive channel is routed through the object, from the cell to a separate location on the object's surface away from the display cells, which can act as the connection point for linking power, signals and touch data to and from the object. Individual cell channels are kept disjoint so as to allow independent control. A distinct surface connection site is also embedded which will hold the channel that attaches to the cell's top electrode. The object is printed on a multimaterial $3 \mathrm{D}$ printer in conductive and insulating PLA.

Spraying phase: the object is sprayed with three coats of material. First, the dielectric layer directly onto the surface using a typical gravity feed airbrush and air compressor. The dielectric layer must cover all the conductive PLA that is intended for EL cells to avoid a short circuit. It must not cover the channel connection sites or the surface connection sites, to allow connection of the power supply. Under a UV light (to allow sight of layering to help ensure coverage) the light-emitting layer is sprayed on the same area as the dielectric. The surface electrode is sprayed to a smaller area than the two layers not to make contact with the channel connection sites, but only with the surface connection site. Spraying is carried out at 40-50psi for the dielectric and emissive layers and 10-20psi for the top electrode (due to lower viscosity). Spraying is carried out at about $30 \mathrm{~cm}$ away from the substrate, for susbtance adhesion, thickness and consistency between attempts. Spraying was carried out at low airflow, in a ventilated area, and low paint flow where possible to increase control.

Lighting up: the power supply (standard EL inverter [30] powered by two $9 \mathrm{~V}$ batteries) is wired with one side attaching to the surface connection site (connecting to the top electrode) and the other attaching to the various connectors to the bottom electrodes via a series of relays for configurability.

Apparatus and Material: we used an Ultimaker S5 3D printer on default settings with a $0.4 \mathrm{~mm}$ nozzle/print core, printing at a $0.15 \mathrm{~mm}$ layer height with conductive filament printed with a $100 \%$ infill to maximise conductivity. We reduced tension in the conductive filament feeder (it is less cohesive than standard PLA) and increased the nozzle temperature to $220^{\circ} \mathrm{C}$ to cope with the properties of the conductive filament. For spray coating we used an Iwata Eclipse hp-cs airbrush with a $0.35 \mathrm{~mm}$ nozzle and an AS186 air compressor. For the base electrode we used commercially available conductive PLA 
infused with carbon black from ProtoPasta [38]. For tests that included masking the base electrode, we sprayed copper-based conductive paint. For the dielectric layer, we used Lumilor's dielectric paint [30], and in the Mobius strip demo Figure 1 (c), a clear lacquer in a spray-can [17]. For the emissive layer we used EL phosphor based paint, though other materials can be used [3, 8, 14, 41, 52]. For the top electrode layer we used poly(3,4-ethylenedioxythiophene):polystyrene sulfonate (PEDOT:PSS) which is an aqueous transparent conductive polymer ink. Our spraying materials were obtained from LumiLor. An additional clear protective layer (lacquer) was used to prime acrylic surfaces and protect the displays.

\section{ProtoSpray enables touch}

In addition to powering the EL cell, the electrodes can serve as capacitance sensors to enable touch sensing. Lacquer layers can be added to the top to create a protective insulating layer and separate the AC current powering the display from the user's touch. We experimented with detecting touch through layers of EL material and lacquer. These showed comparable capacitive sensing capabilities to channels with no coating. We do not explore this functionality further as this is already thoroughly covered in previous work such as Capricate [43] and thus not a new contribution. Stretchis [52] detects change in capacitance by monitoring a conductive channel for signal, as it is not directly possible when the AC power is on. Alternatively the power and sensing can be time-multiplexed over the same embedded channels but at different times, at a frequency above human visual perception to eliminate visual flickering, as in PrintScreen [34].

\section{COMPARING MASKING TECHNIQUES}

Our investigation on the feasibility of ProtoSpray starts by looking at a single electrode on a flat surface. As we want to replace sprayed base electrodes with conductive $3 \mathrm{D}$ printed channels, our goal is to compare masking on different layers to establish that shaping the cell's base electrode could provide comparable display quality to masking other layers.

\section{Test 1: comparing masking layers}

Summary. How does masking different layers of the spraying process affect the precision of cell shapes? We found that masking the top or bottom electrode layer is more precise than masking the light-emitting layer.

Comparison samples. To test the effectiveness of masking different layers, we produced three sample EL cells with either the base electrode, light-emitting layer or top electrode masked (Figure 4). Further details for replicability are included in the annex to this paper.

Analysis. We used the perimeter-to-area ratio of each of the cells as a metric to determine which of the samples had the most precise edge. We used a pixel counting tool to measure the distance around the lit up area of the cell using the original stencil to provide scale.

Results. The stencil had a perimeter of $247.6 \mathrm{~mm}$ and an area of $1547 \mathrm{~mm}^{2}$ giving an edge roughness parameter of 0.172 . The masked base electrode gave a roughness parameter of 0.241 . A masked emissive layer gave a reading of 0.281 and the masked top electrode measured at 0.238 . This information gives the masking of the emissive layer as the least effective in terms of roughness. The results suggest that we would have most success in masking the top electrode although the bottom electrode is comparable. Visually, masking the bottom electrode gives the best results. Additionally, masking separate layers presents different fabrication considerations. Masking the bottom layer requires post-process sanding. Masking the top layer, over paint, requires longer drying times ( +1 hour) to ensure not having issues with displacement from the stencil.
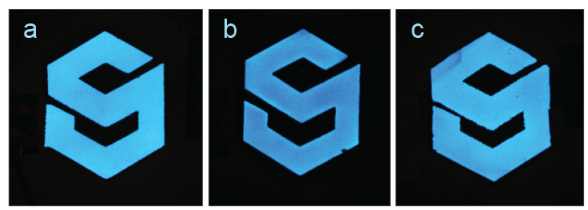

Figure 4. Test 1: three EL cells with the same mask used on different layers: a) base electrode masked; b) light-emitting layer masked; c) surface electrode masked.

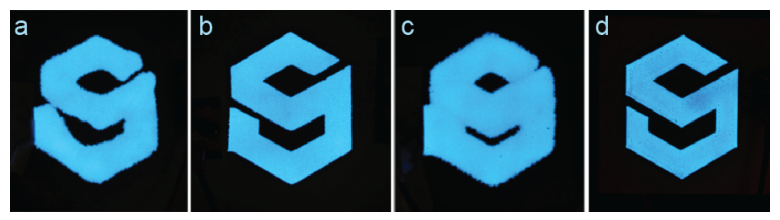

Figure 5. Test 2: four EL cells sprayed onto flat substrates using techniques to define cell shapes on the base electrode: a) hand masking; b) contact masking; c) shadow masking; d) 3D printer-shaped cells.

\section{Test 2: comparing masking methods}

Summary. How does the precision of different methods of defining cell shape compare? We found the process of eliminating masking as precise as contact masking, and better than shadow masking or hand masking.

Comparison samples. We investigated masking the base electrode to explore the scope of later defining cells without masking. We compared fidelity of the final EL cell to the intended design and the precision of edges through different types of masking, to determine an effective method. We compared baseline 'hand masking' performed without stencil; 'Contact masking'; 'Shadow masking'; and ProtoSpray (Figure 5).

Analysis. As in Test 1, we test the fidelity of the mask to the intended shape and the precision of mask edges via image analysis. We remove the area covered by the stencil from the EL cell area. This gives a cell overspray 'halo' which we can compare to the original stencil as an overlap percentage.

Results. The base stencil had a perimeter-by-area roughness measurement of 0.172 and none of the samples produced undercoverage compared to the design. We recorded measurements of 0.391 for the hand masked cell, 0.241 for the copper contact mask, 0.242 for the shadow mask and 0.246 for the 3D printed-shaped cell. The shadow mask is clearly a poor rendition. The hand masked cell covered an extra $10 \%$ of the intended area. The contact masked $2 \%$, the shadow masked $35 \%$ and the 3D printer-shaped cell $0.1 \%$. The contact masked cell and the 3D printer-shaped cell have the best representations of the original design by area, with the shadow mask being the worst. Looking at both measures, shaping the cell using the 3D printer performs better than hand masking and shadow masking, and is similar to contact masking while removing negative aspects of contact masking such as sanding. 


\section{EXPLORING SURFACE TOPOLOGY}

Having demonstrated ProtoSpray on flat surfaces, we tested more complex shapes, particularly non-smooth surfaces (angle and curves) and surfaces with zero-crossing. We chose these as they can be affect by spraying in two ways: (1) material sprayed from an airbrush is concentrated in the center of the plume. Spraying perpendicularly to a surface is most effective for an even coating. Surfaces with high 'shape resolution' [40] (e.g. significant curvature or multiple zero-crossings) thus create challenges when spraying; (2) spraying is challenged by discrete angular intersections of surfaces, as it is difficult to spray a single coherent surface across a sharp edge.

\section{Test 3: angular and curved surfaces}

Summary. What shaped topologies are supported by the ProtoSpray process of mixed media printing of substrates for subsequent spraying of EL cells? We found that a range of angular shapes and curves in one and two dimensions support fully functioning EL cells being produced.

Comparison samples. We fabricated five objects each with a single EL cell on an irregular surface (Figure 6). The first three (a-c) incorporated a triangular raised section of steepening peak angle to explore the limits of 'sharpness' on the ProtoSpray method. We produced cells with 90 degree, 60 degree and 30 degree angles. We were limited in producing a narrower angle due to 3D printer resolution. The concave joins at the bottom of the triangular prisms were smoothed out so that we could isolate issues around the convex point at the top of the triangle. The other samples were similar EL strips. The first of these (d) is a plane that curves in a single dimension. The second (e) is a plane that curves in two dimensions in the shape of a section of a sphere. The size of the EL strips was chosen to be of a similar area to those in previous tests (80mm-120mm long, $10 \mathrm{~mm}$ wide) for consistent spray coverage and reduced likelihood of printing faults. All samples were printed and sprayed with EL cells facing upwards to ensure even drying and consistent slicing from the 3D printing software.

Analysis and results. Of particular interest was whether cells worked over the peak angle or curvature being tested. In this experiment we are looking at whether the sample worked for the full length of the EL cell or if it had issues with consistent luminescence along its length, and failure at the angle peak. We were also looking to see if the ProtoSpray approach was significantly constrained by curved surfaces in one and two dimensions. We found that all five of the shapes allowed sufficient coverage of sprayed materials to produce a functional, fully lit EL cell.

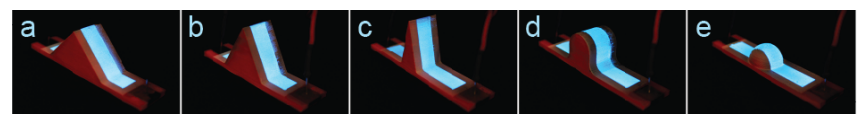

Figure 6. Test 3: EL cells on different topologies. Rectangular cells are printed/sprayed over different angles (90 degrees, 60 degrees and 30 degrees), a curved semicircular prism and a hemispherical surface

\section{Test 4: Continuous curved surfaces}

Summary. What degree of zero-crossing prevents cohesive layering? We found that 3D printed curvature spanning multiple zero crossings is unreliable for consistency of spraying EL cells at multiple scales.

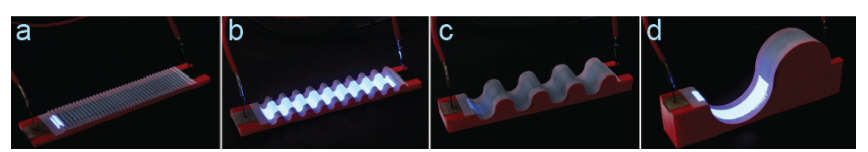

Figure 7. Test 4: four EL cells on curved topologies of regularly incrementing size, lit up with different levels of success.

Comparison samples. We observe the extent to which ProtoSpray can cope with zero-crossing curvature at different scales, specifically to understand how the size of curves affect the angle of 'access' from the airbrush and prevent cohesive layering. We compared four surfaces, each structured as a wave with circular curvature (Figure 7). We chose this shape as a replicable pattern at multiple scales, with regular curvature, where the surface was not all sprayable from a perpendicular angle due to vertical gradients. We made four samples with half-wavelength measurements of $1 \mathrm{~mm}$ (a), $4 \mathrm{~mm}$ (b), $10 \mathrm{~mm}$ (c) and 40mm (d). These were made to the same length, so had 40, 10, 4 and 1 peaks respectively, each with a total surface length of $251.4 \mathrm{~mm}$. Each sample was printed using embedded printed conductive base electrodes to create a strip EL cell $10 \mathrm{~mm}$ across, to ensure an even coverage relative to airbrush plume size. The designs were made with smoothed corners between the electrode contact points and the curvature section to ensure no angular boundary risking the failure of the cell.

Analysis. Success was defined by whether a cell covered at least one convex and concave part of the curved surface and secondarily by the length of the surface that was lit up.

Results. All samples produced (Figure 7) were at least partial failures. The two smaller samples (a) and (b) had at least one full wave length of illuminated cell, demonstrating the method coping with zero-crossings (and non-perpendicular spraying). However the larger samples did not produce cells running a full wave length and none of the samples produced the full length of the EL cell with full illumination. Sample (d) had a length of $125.7 \mathrm{~mm}$ lit up (measured along the surface of the EL cell. Sample (c) had $7.9 \mathrm{~mm}$ lit up, (b) gave $238.8 \mathrm{~mm}$ lit up and (a) gave $3.1 \mathrm{~mm}$ lit up. Since all 4 cells lit up from side of the top electrode connection point we are able to deduce that the base electrode was cohesive and delivered charge along its length, and that the surface electrode was the one that failed. The PEDOT:PSS used for the top electrode has lower viscosity than the other sprayed materials and this is likely the reason it did not achieve full coverage in the same topologies and areas. We conclude that the process has the potential to work on some continuous curved topologies when perpendicular spraying is not possible, but it struggles with zero-crossings.

\section{UNDERSTANDING PRINTER CONSIDERATIONS}

The final step is to understand challenges arising when integrating 3D printed and spray as a single device. We focus on spray orientation and 3D printer resolution.

\section{Test 5: Spray orientation}

Summary. Does spraying orientation affect cell functionality, either due to gravity or drying? We found that lower viscosity material can drip, potentially disrupting electrical function.

Comparison samples. We sprayed 3 samples of PEDOT:PSS and 3 of copper-based conductive paint onto identical primed acrylic panes, chosen for their smooth surface. A control sample was sprayed vertically downwards, one was sprayed 
horizontally at 90 degrees to maximise dripping due to gravity, and the third was sprayed upwards at a 135 degree angle to maximise the effect of gravity on airbrush particles. We chose the two materials as they are conductive and we can test resistance across them. The copper paint used has similar viscosity to the materials we used in other experiments for the emissive layer and dielectric layer. In contrast, PEDOT:PSS is aqueous and far less viscous. The substrates were left at the same angle while drying with minimal airflow over the surface. We chose to spray a controlled amount to see what the distribution of paint was in a 'normal' environment, with 50\% deposition overlap for a single cohesive layer and the airbrush $20 \mathrm{~cm}$ away, to build a continuously conductive surface. The airbrush was held perpendicularly to the substrate.

Analysis. As a non-destructive metric for thickness, we tested the resistances of the thin film conductive surfaces along a horizontal line. A thicker conductive layer provides greater resistance, a thinner film provides less. Any settling of particles in the plume of the airbrush or while drying will increase the thickness of the paint on the substrate and so will measure a lower resistance. Due to the overlapping patterning of spraying, it is likely that a thicker layer of paint would occur in the middle of the test panes, with a thinner layer towards the edges. We were able to measure the approximate spread of this using our vertically sprayed control sample. We took discrete resistance readings at regular $10 \mathrm{~mm}$ intervals along each of the six panes.

Results. Figure 8 shows that for copper there are higher resistances in the top and bottom. This is likely because uniform coverage with the airbrush involves more overlap on central areas. As a result, measuring a gradient of lowering resistance towards the bottom of the pane will not necessarily indicate gravitational influence on the material. Asymmetry in resistance readings between top and bottom measurements in turn could indicate inconsistencies in paint thickness due to gravitational effect. This can be seen in the 135 degree angle measurements ( $21.4 \mathrm{k} \Omega$ vs $10.9 \mathrm{k} \Omega$ ) and to a lesser extent in the 90 degree angle measurements $(85.7 k \Omega$ vs $66.3 k \Omega)$. For the PEDOT:PSS the trend changes: we measured similar resistances on top and bottom and observed visible dripping. Uneven material on steeper gradients can affect light consistency, reliable fabrication and create design constraints. Our results suggest that this is not significant for heavier materials, and so ProtoSpray is less hindered by these constraints.
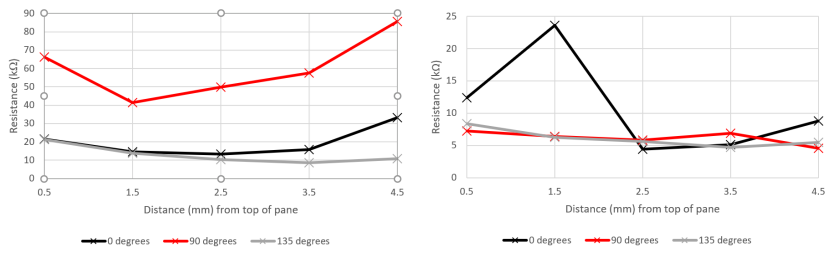

Figure 8. Test 5: Horizontal resistance readings of different angles for copper paint (left) and PEDOT:PSS (right).

\section{Test 6: 3D printer resolution}

Summary. Does printing resolution and post-process treatment improve the quality of the EL cell? Yes, a higher print resolution produces a more usable EL cell, however sanding does not appear to improve EL cell quality.
Comparison samples. 3D printed PLA has inherent porosity and the surface has irregular 'contouring' from extruding in layers. This effect is exacerbated by a curved 3D printed surface due to voxel rasterisation. A more irregular surface is more likely to create areas which are not evenly covered by all three sprayed layers that form the EL cell. Sharp corners in the base electrode can cause parts of the surface to be sheltered from the airbrush plume. We investigate whether these issues can be mitigated by post production treatment such as sanding. We are thus interested in the inherent tradeoff between print time and surface quality. We printed 3 domes with an EL cell. We used a curved surface as the layering of the 3D printer creates the most ridges and sharp angles on a curve (relatively smooth on a flat surface). Each object had a single channel. We printed: (1) a $0.15 \mathrm{~mm}$ layer height, as an unsanded control object; (2) a $0.15 \mathrm{~mm}$ layer height version thoroughly sanded to give a smooth finish; and (3) $0.06 \mathrm{~mm}$ layer height that is unsanded (the maximum resolution of the $3 \mathrm{D}$ printer). All other print settings and spray considerations were kept constant. The sanding was carried out with 500 grit sandpaper followed by 1500 grit sandpaper.

Analysis. We counted the number of visible faults not illuminated within the EL cells. We did this by taking images that we first desaturated and downsampled to binary so that the light emitting areas could be measured. Applying ImageJ's particle analysis tool [19] to the selected area, we counted the number of faults (areas not emitting light) and their sizes.

Results. For the unsanded print at standard print settings (0.15mm layer height), 271 faults were counted with a mean size of $2.10 \mathrm{~mm}^{2}$. For the sanded print, 496 faults were counted with a mean size of $1.54 \mathrm{~mm}^{2}$. The unsanded version, printed at the highest quality of layering $(0.06 \mathrm{~mm}$ layer height $)$ had 78 faults with a mean size of $0.71 \mathrm{~mm}^{2}$. Printing the model at a finer layer height produced far fewer faults and those faults were smaller. But sanding increased the number of faults, although they were smaller on average. Sanding was expected to reduce the roughness of the print surface and so decrease the number of faults measured in the EL cell. Due to PLA's low melting temperature, it is possible that the sanding damaged the surface. The high quality unsanded print took 11.5 hours to print compared to 5.5 hours for the others. This test highlights the payoff between fabrication speed and quality of output.

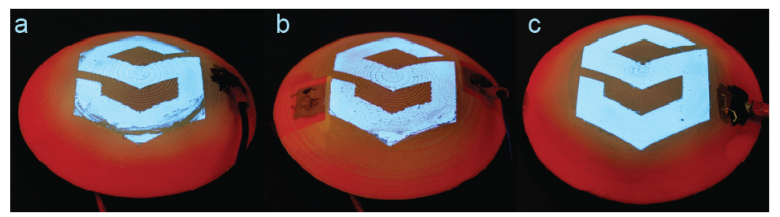

Figure 9. Test 6: Cells printed on different scales of 3D printed resolutions. a) unsanded on a $0.15 \mathrm{~mm}$ layer height; b) sanded on a $0.15 \mathrm{~mm}$ layer height; c) unsanded on a $0.06 \mathrm{~mm}$ layer height

\section{DEMONSTRATORS}

Our prototypes demonstrate applications of ProtoSpray, the benefits of using 3D printed channelling, as well as range of topologies showing that we can go beyond current techniques.

\section{Segment and matrix displays}

We start with a 7-segment display taking advantage of conductive channelling (Figure $10(a, b)$ ). The demonstration shows 
the potential for creation of usable and customisable luminescent widgets through ProtoSpray. The channelling allows for a design, without needing to accommodate conductive surface traces or for masking electrode contact points. While we have manually routed these conductive channels within the 3D printed object, for complex displays we could draw on existing 3D network-on-chip channelling algorithms to optimise energy consumption and channel layout while taking into account spacing constraints [5]. Such algorithms can take into account preferred channel orientation, which in our case could align with printer layers to improve conductivity.

The second example is a $6 \times 6$ pixel matrix display, shown in Figure 10, which demonstrates a display with spacing between cells (channels $2 \mathrm{~mm}$ apart, cells $4 \mathrm{~mm}$ square). This demonstration shows the potential for fully configurable information displays: the matrix allows each pixel to be individually activated rather than being a passive matrix display as in other methods [34]. We are able to address each pixel individually as a direct benefit of the ProtoSpray process via 3D printing a substrate with embedded conductive channels that each form an attachment under the display, through the object rather than on it's surface, thereby not obscuring the matrix display or requiring further spaced apart pixels for conductive traces.

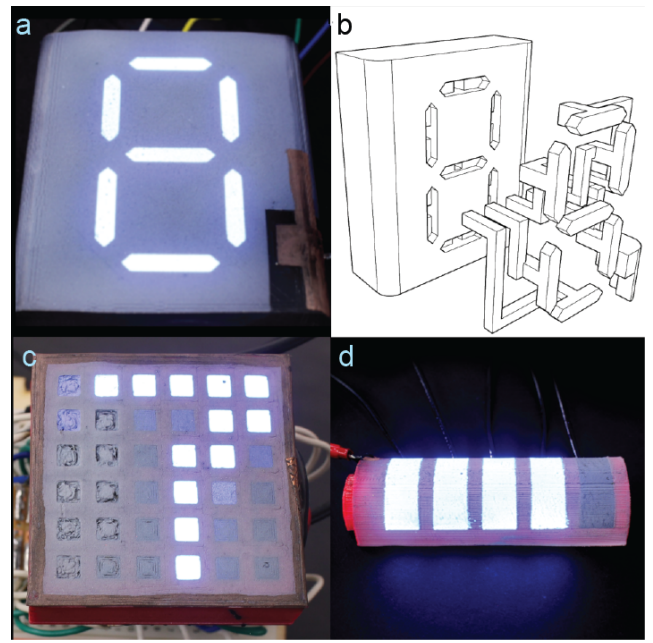

Figure 10. a) The demonstration of a configurable 7-segment display using ProtoSpray; b) A diagram of the design for the channelling that is required for the 7-segment display, showing channels that would otherwise overlap on a 2D surface; c) A 6x6 configurable matrix display created using the ProtoSpray process. Pixels are $4 \mathrm{mmx} 4 \mathrm{~mm}$ squares with $2 \mathrm{~mm}$ in-between them; d) ProtoSpray on a curved surface to create an interactive power level indicator, 3D printed in the shape of a battery.

\section{Four complex curvatures}

To demonstrate ProtoSpray's applicability to irregular designs we created four examples of dynamic displays with multiple electrodes on non-flat surfaces: a cube (Figure 1 (a)), a cylinder (Figure $10(\mathrm{~d})$ ), a dome and a Mobius strip (Figure 1 (b,c)). The cube demonstrates that sharp angles are not an obstacle to creating displays. The cylinder uses the benefits of conductive channels to obscure connection points to base electrodes under the object both for the spraying process and usage. This would not be possible if the base electrodes were applied as hydro printed patterns or prefabricated screen printed cells.
The dome demonstrates a curvature in two planes that is impossible to create through deformation of a flat surface such as using a prefabricated EL cell. It also further demonstrates benefits of the channelling in ProtoSpray compared to traditional EL layering methods: central cells are enclosed by the outer cells. Creating such an object through other fabrication processes without the use of conductive channels (such as layering an ink-based base electrode through hydro printing) would require electrodes crossing other outer cells causing short circuits (or complicated bridging requiring multiple additional layers and further risks of failure).

The Mobius strip has 7 non-flat segmented EL cells in the shape of arrows that can light up sequentially, providing a visual indication of the movement around the strip along its single side. This demonstrator shows the benefits of channelling for the fabrication process as the electrode connection points are obscured from creating short circuits by the bulk of the object, even though they are each at different angles on the strip. It also demonstrates the benefit of spraying EL material as the materials were each applied in one go despite curved EL cells with normals in all directions. The channelling provides accuracy of base electrodes, without the need for masking, which is a challenging and highly skill dependent process on such a topologically complex object. Channelling is necessary to reduce the surface area taken up by surface electrodes and to simplify electrode connection points to areas on the Mobius strip that are away from EL cells but still attached. Spraying allowed the coating of each of the layers required for EL cells in a single pass, despite the complicated base object topology, and having EL cells in 7 planes dependent on their position on the Mobius strip. Additionally, material that was too thinly applied to ensure a working prototype could be corrected by adding more material, with no distortion.

\section{DISCUSSION AND LIMITATIONS}

\section{Summary of findings}

Through our tests and demonstrations we have characterised and determined appropriate parameters for the ProtoSpray fabrication process. We have summarised our findings at the beginning of each test to help researchers and designers use them. We have not yet built a platform that integrates 3D printing with automated airbrushing (see our vision in Figure 11), but our findings provide the detail necessary to demonstrate the feasibility of our approach. These including hardware and software design constraints such as identifying when fabrication processes may be approaching failure tolerances.

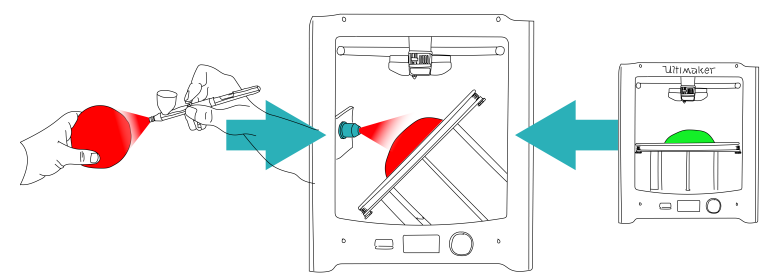

Figure 11. Vision for a hybrid spraying and extrusion based machine.

Overall, we are excited by the dissemination of 3D printing technology but feel that there are still intrinsic limitations. This is particularly true when it comes to accuracy in materials that can be manufactured. We envision a machine (Figure 11) that 
can address these issues, while allowing printing of regular objects due to the combination of spraying and extrusion. Such a machine would combine 3D printing and automated spraying so that fabrication demonstrated in this paper could be fully automated, and printing and spraying could be interlinked and alternated to achieve a broader range of topologies and shapes for EL cells.

\section{Fabrication speed}

3D printing is not a fast fabrication process, and many users run jobs overnight or in parallel to optimise build time. Our experience is that 3D printing speed remains the bottleneck in producing ProtoSpray objects. However, spraying displays can also be time intensive, and so there are factors to consider to optimise the fabrication process. It takes under 2 hours to spray the EL layers with our current set up. This time is not significantly increased by spraying multiple objects simultaneously, as it does not take much additional effort to spray the same layer across multiple cells. Key factors include any contact masking required, cleaning of equipment, and preparing materials and equipment. ProtoSpray inherently presents two features that reduce spraying time: 3D printing the bottom electrode rather than masking reduces complexity of production and overall spraying times; and electrode attachment time is reduced by directly inserting pin electrodes. Practical spraying considerations include drying times and cleaning the airbrush between each layer to avoid clogging. Prototyping time could be further minimised with the use of automated spray nozzles and mechanical nozzle cleaning.

\section{Health and safety considerations}

While 3D printing requires a fabrication space with some level of operator risk, the introduction of spraying and electrical prototyping demand further consideration of health and safety parameters. Protospray introduces processes for material management, protection against inhalation, cleaning and storage requirements, and electrical safety for operating prototypes at higher voltages. Airbrushes eject aerosols into the environment, and it is important to consider the effects of inhalation. Droplet sizes created by airbrushes are especially important, as the degree of aerosol particle retention in the respiratory systems is a function of particle radius. For our work, we have used high quality respirators (standard P3 in Europe, P100 in the US) as well as a small bench top fume hood with both HEPA and Carbon filters as an additional safety measure against ambient distribution of particles and fumes. Such protective equipment is important to ensure health and safety of end-users using ProtoSpray and others within the same space. The power used in this circuit has a low current reducing the danger of shocks (as discussed in [34]), and the risk is further reduced by careful application of lacquer.

\section{Limit in the electrode dimensions}

In the process of developing ProtoSpray we also tested the effect of channel width, length and print orientation on resistance, the impact of cell size on resolution and the effect of crosstalk between conductive channels relative to their location (see supporting annex). Although it is relevant, the work on resistances is not new, e.g. resistance decreases exponentially relative to channel width, proportional to length and the direction of print has an effect $[22,43]$.

\section{Note on electrode attachment}

Connecting the ProtoSprayed objects to a microcontroller also required an exploratory design process. Alongside using channelling to create easy connection sites with regular ordered channels, we looked at wire-to-PLA connections. We found that a directly inserted pin into a conductive PLA cavity is an efficient method of electrode connection without significant change to the original object. We tested several methods (Figure 12) for resistance, as well as looking at ease of application. These methods included clipping(1.5k $\Omega$ ) (a), sticking with epoxy, copper and copper connection paint $(2.9 \mathrm{k} \Omega$ and $1.8 \mathrm{k} \Omega$ for painted/sprayed connection paint respectively) $(b, d)$ and embedding (1.4k $\Omega$ ) (c). All connection methods have different design constraints on the final object and we found that insertion was both the fastest/most reliable connector and had the least visible impact on the final object design.

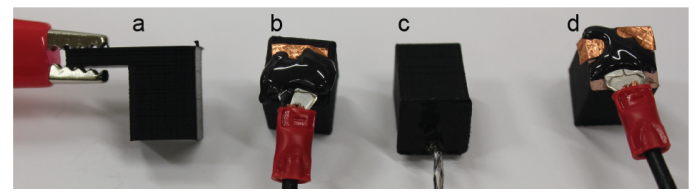

Figure 12. Attachments of circuit to 3D printed conductive objects. a) printed lip with clip attachment; b) painted copper - spade attachment with epoxy fixing; c) printed guide hole with embedded pin; d) sprayed copper - spade attachment with epoxy fixing.

\section{Fabrication lessons and failures}

The thickness of the layers of paint is able to vary significantly and still produce functional EL cells, making this method suitable for user-based airbrushing. Although it had a small effect on the brightness and consistency of the light produced, we found that the dielectric and emissive layers could be applied very thinly or thickly as long as there were no holes. Application of material was more effective and reliable in two thin coats to avoid displacement of previous paint or cracking, and this was especially the case with the low viscosity PEDOT:PSS, which was responsible for most of our failed prints due to dripping or inhomogeneous layers. The dielectric and emissive materials are viscous suspensions and clog up the airbrush, requiring shaking and high pressure in order to be sprayed (40-50 PSI). In some cases, passing a current through the $3 \mathrm{D}$ print caused some minor melting of contact points between conductive PLA and metal wires, although there were no cases where this caused inherent damage to the object.

For post-processing, we used thick then fine grit sandpaper to give the best results, and used a file in stubborn cases. Sanding wasn't necessary, but increased the reliability of the process and likeliness to produce a working object. Unsanded objects risked insufficient dielectric and emissive coverage and weak application of the PEDOT:PSS for a continuous conductive surface. Additionally, objects printed with any significant underextrusion (often due to experimental nature of conductive PLA) were rejected due to porosity increasing the potential for inhomogenous layers of dielectric/emissive and so increasing the potential for shorts. In some cases, copper paint was applied to the connection between the conductive PLA top electrode connection sites and the PEDOT:PSS top electrode to improve the reliability of the electrical connection. For more complex topologies, the clear lacquer was used as the dielectric layer due to improved impermeability, reduced risk of 


\begin{tabular}{||rll||}
\hline Test & Question & Result \\
\hline \hline 1 & Layers to mask & Either electrode \\
\hline 2 & Define cell shape & 3D printed electrode \\
\hline 3 & Surface shape tolerance & range of angles/curves \\
\hline 4 & Topology robustness & Zero crossings \\
\hline 5 & Spray orientation & Viscosity dependent \\
\hline 6 & 3D printer resolution & Reduces faults \\
\hline
\end{tabular}

Table 1. Summary of test results

electric shocks and avoidance of shorts. Objects produced had tolerance to scratching but were susceptible to water damage without sufficient lamination layers. Adhesion between plastics and layers was not observed to be a problem at any stage but more formal testing would need to be carried out, beyond the scope of this paper. If fabricating the prototype failed due to electrical shorts or cross-talk between base electrodes, the process had to be restarted from scratch.

\section{Limitations in methods}

The tests carried out are designed to demonstrate the feasibility of functionality of the ProtoSpray process. We conducted a single repetition of each successful test, as this was sufficient to show the workablity of the process. In addition, there was significant noise and a range of unmeasurable user based factors in the process through the sanding and handheld spraying. The samples were made and experimented on by a researcher who worked on the project for several months. Future work is required to measure the robustness of end users' ability to replicate these results. Issues with usability are only the case when the process is carried out manually (see our vision which would remove the noise altogether). Additionally, this paper's methodology is structured to cover breadth of potential applications of the process. As a result, included tests demonstrate the range of possible applications, but there are still significant further areas (such as more complex topologies) to be explored. Further work could look at concave versions of the topologies explored, objects with holes in them and geometries that enclose themselves. There are also further questions to explore in the context of scalability and size. In particular, the benefits of channelling rely on a single continuous top electrode for all cells which will provide difficulty in scaling. In this paper we focus on producing smaller cells and constraints in this context. We establish that layer height and 3D printer resolution are key issues for smaller EL cells. For larger EL cells however one must consider other factors such as printer bed size, electrical power and material implications.

\section{Materials future work}

We have used off-the-shelf materials to spray EL display layers. All the functional materials that we have used - conductive, dielectric and electroluminescent - are active areas of research, and new methods of deposition are also being actively studied in manufacturing and the wider scientific literature. New forms of highly conductive 3D printing material are available, including for example enhanced carbon-based filament [22, 38], or nano-silver paste exploited by Voxel8 [48]. Increased conductivity could further enhance energy efficiency, however the thickness of the dielectric and EL layers are more critical than channel conductivity for electrical efficiency of the display.
We could in principle use a thermoplastic such as PLA as a dielectric material, further reducing the required number of sprayed layers. To explore this idea, we extruded the thinnest layer $(0.06 \mathrm{~mm})$ of PLA possible on the Ultimaker S5 as dielectric on top of an embedded electrode, and oversprayed EL and top electrode layers. The result (Figure 13) shows this working, but with poor consistency and overall performance. Increasing 3D printer resolution may support this approach

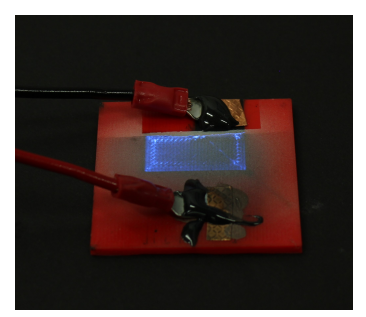

Figure 13. El cell shaped without masking, using a conductive PLA base electrode with a $0.06 \mathrm{~mm}$ extruded insulating PLA as dielectric layer - only emissive and surface electrode layers are sprayed on the object. in the future with potential for extruding smoother surfaces and thinner layers. However, we will not likely be able to extrude transparent polymers for top electrodes due to material properties. Considering thin films, it may be possible to further revise our 'recipe' to introduce semiconductors more closely aligned with OLEDs, QLEDs or Light-emitting Electrochemical Cells (LECs). To operate these thin films at low voltages, we would need more precise deposition processes than airbrush spraying, such as evaporation coating.

\section{CONCLUSION}

This paper contributes ProtoSpray, a novel fabrication method for creating arbitrary shaped objects with EL displays embedded into them. We have introduced spraying as a method for layering thin-film EL displays onto irregular surfaces and conducted a series of tests to analyse its feasibility (see Table 1). We have also introduced the use of conductive channelling for producing objects with EL display elements. We have combined these through 3D printing with insulating and conductive PLA and then sprayed-on surfaces to create interactive custom displays. These combined fabrication tools allow the creation of custom displays using EL materials with irregular topologies which goes beyond previous work. We hope our work can be an inspiration for the HCI community, which has been thriving in recent years in producing examples of interactive devices that go beyond rectangular shapes. Our technique can be used to create a large range of new interactive devices with exotic form factors and has potential applications in many domains such as handheld devices and interactive controllers, urban displays such as signage, ambient displays, and many other areas that could benefit from democratised fabrication of displays directly embedded onto 3D printed objects.

\section{ACKNOWLEDGMENTS}

This work was supported by the Engineering and Physical Sciences Research Council (EP/M021882/1 and EP/P004342/1) and the Faculty of Engineering of the University of Bristol.

\section{REFERENCES}

1. Alaa Abdellah, Kulpreet Singh Virdi, Robert Meier, Markus Döblinger, Peter Müller-Buschbaum, Christina Scheu, Paolo Lugli, and Giuseppe Scarpa. 2012. Successive spray deposition of P3HT/PCBM organic photoactive layers: material composition and device characteristics. Advanced Functional Materials 22, 19 
(2012), 4078-4086. DOI :

http://dx.doi .org/10.1002/adfm. 201200548

2. Eric Akaoka, Tim Ginn, and Roel Vertegaal. 2010. DisplayObjects: prototyping functional physical interfaces on 3d styrofoam, paper or cardboard models. In Proceedings of the fourth international conference on Tangible, embedded, and embodied interaction. ACM, 49-56. DOI : http://dx. doi .org/10.1145/1709886. 1709897

3. Mariya Petrova Aleksandrova. 2012. Improvement of the electrical characteristics of polymer electroluminescent structures by using spray-coating technology. Journal of Coatings Technology and Research 9, 2 (2012), 157-161. DOI :http://dx.doi.org/10.1007/s11998-009-9220-2

4. Jason Alexander, Andrés Lucero, and Sriram Subramanian. 2012. Tilt displays: designing display surfaces with multi-axis tilting and actuation. Proceedings of the 14th international conference on Human-computer interaction with mobile devices and services (2012), 161-170. DOI :

http://dx.doi.org/10.1145/2371574.2371600

5. Abdul Quaiyum Ansari, Mohammad Rashid Ansari, and Mohammad Ayoub Khan. 2016. Modified quadrant-based routing algorithm for 3D Torus Network-on-Chip architecture. Perspectives in Science 8 (2016), 718-721. DOI : http://dx.doi.org/10.1016/j.pisc.2016.06.069

6. Thomas Auzinger, Wolfgang Heidrich, and Bernd Bickel. 2018. Computational design of nanostructural color for additive manufacturing. ACM Transactions on Graphics (TOG) 37, 4 (2018), 159. DOI :

http://dx.doi.org/10.1145/3197517.3201376

7. Hrvoje Benko, Andrew D Wilson, and Ravin

Balakrishnan. 2008. Sphere: multi-touch interactions on a spherical display. Proceedings of the 21st annual ACM symposium on User interface software and technology (2008), 77-86. DOI :

http://dx.doi.org/10.1145/1449715. 1449729

8. Jayesh Bharathan and Yang Yang. 1998. Polymer electroluminescent devices processed by inkjet printing: I. Polymer light-emitting logo. Applied Physics Letters 72, 21 (1998), 2660-2662. DOI :

http://dx.doi.org/10.1063/1.121090

9. Eric Brockmeyer, Ivan Poupyrev, and Scott Hudson. 2013. PAPILLON: designing curved display surfaces with printed optics. Proceedings of the 26th annual ACM symposium on User interface software and technology (2013), 457-462. DOI :

http://dx.doi.org/10.1145/2501988.2502027

10. Aubrey L Dyer, Emily J Thompson, and John R Reynolds. 2011. Completing the color palette with spray-processable polymer electrochromics. ACS applied materials \& interfaces 3, 6 (2011), 1787-1795. DOI:

http://dx.doi.org/10.1021/am200040p

11. Joshua Elsdon and Yiannis Demiris. 2017. Assisted painting of 3D structures using shared control with a hand-held robot. In 2017 IEEE International Conference on Robotics and Automation (ICRA). IEEE, 4891-4897. DOI : http://dx. doi .org/10.1109/ICRA.2017.7989566
12. Fernando Ely, Agatha Matsumoto, Bram Zoetebier, Valdirene S Peressinotto, Marcelo Kioshi Hirata, Douglas A de Sousa, and Rubens Maciel. 2014. Handheld and automated ultrasonic spray deposition of conductive PEDOT: PSS films and their application in AC EL devices. Organic electronics 15, 5 (2014), 1062-1070. DOI :http://dx.doi.org/10.1016/j.orgel.2014.02.022

13. Aniello Falco, Mattia Petrelli, Emanuele Bezzeccheri, Ahmed Abdelhalim, and Paolo Lugli. 2016. Towards 3D-printed organic electronics: Planarization and spray-deposition of functional layers onto 3D-printed objects. Organic electronics 39 (2016), 340-347. DOI : http://dx.doi.org/10.1016/j.orgel.2016.10.027

14. Katsuhiko Fujita, Takamasa Ishikawa, and Tetsuo Tsutsui. 2003. Separate-coating and layer-by-layer deposition of polymer emitting materials by the spray deposition. Mol. Cryst. Liq. Cryst. 405, 1 (2003), 83-88. DOI : http://dx.doi.org/10.1080/15421400390264180

15. Daniel Groeger and Jürgen Steimle. 2018. ObjectSkin: augmenting everyday objects with hydroprinted touch sensors and displays. Proceedings of the ACM on Interactive, Mobile, Wearable and Ubiquitous Technologies 1, 4 (2018), 134. DOI: http://dx.doi.org/10.1145/3161165

16. Ghazaleh Haghiashtiani, Ed Habtour, Sung-Hyun Park, Frank Gardea, and Michael C McAlpine. 2018. 3D printed electrically-driven soft actuators. Extreme Mechanics Letters 21 (2018), 1-8. DOI :

http://dx.doi.org/10.1016/j.eml .2018.02.002

17. Halfords. 2019. Clear lacquer: https://www.halfords.com/motoring/paints-bodyrepair/car-spray-paints/halfords-clear-lacquer-500ml. Last accessed September 2019. (2019).

18. JC Hitt, JP Bender, and JF Wager. 2000. Thin-film electroluminescent device physics modeling. Critical reviews in solid state and materials sciences 25, 1 (2000), 29-85. DOI:

http://dx.doi .org/10.1080/10408430091149178

19. ImageJ. 2019. Image processing tool in Java: https://imagej.nih.gov/ij/ Last accessed September 2019. (2019).

20. Yoshio Ishiguro and Ivan Poupyrev. 2014. 3D printed interactive speakers. Proceedings of the SIGCHI Conference on Human Factors in Computing Systems (2014), 1733-1742. DOI : http://dx.doi.org/10.1145/2556288.2557046

21. Yuhua Jin, Isabel Qamar, Michael Wessely, Aradhana Adhikari, Katarina Bulovic, Parinya Punpongsanon, and Stefanie Mueller. 2019. Photo-Chromeleon: Re-Programmable Multi-Color Textures Using Photochromic Dyes. In Proceedings of the 32nd Annual ACM Symposium on User Interface Software and Technology. ACM, 701-712. DOI : http://dx.doi.org/10.1145/3332165.3347905 
22. Umme Kalsoom, Pavel N Nesterenko, and Brett Paull. 2016. Recent developments in 3D printable composite materials. RSC Advances 6, 65 (2016), 60355-60371. DOI : http://dx.doi .org/10.1039/C6RA11334F

23. Hee-Dong Kim, Kyeong Heon Kim, Su Jin Kim, and Tae Geun Kim. 2015. Fabrication of conducting-filament-embedded indium tin oxide electrodes: application to lateral-type gallium nitride light-emitting diodes. Optics Express 23, 22 (2015), 28775-28783. DOI :

http://dx.doi.org/10.1364/OE.23.028775

24. Konstantin Klamka and Raimund Dachselt. 2017. IllumiPaper: Illuminated interactive paper. Proceedings of the 2017 CHI Conference on Human Factors in Computing Systems (2017), 5605-5618. DOI : http://dx.doi.org/10.1145/3025453.3025525

25. Yong Lin Kong, Ian A Tamargo, Hyoungsoo Kim, Blake N Johnson, Maneesh K Gupta, Tae-Wook Koh, Huai-An Chin, Daniel A Steingart, Barry P Rand, and Michael C McAlpine. 2014. 3D printed quantum dot light-emitting diodes. Nano letters 14, 12 (2014), 7017-7023. DOI : http://dx.doi .org/10.1021/n15033292

26. Stacey Kuznetsov, Piyum Fernando, Emily Ritter, Cassandra Barrett, Jennifer Weiler, and Marissa Rohr. 2018. Screenprinting and TEI: Supporting Engagement with STEAM through DIY Fabrication of Smart Materials. Proceedings of the Twelfth International Conference on Tangible, Embedded, and Embodied Interaction (2018), 211-220. DOI :

http://dx.doi.org/10.1145/3173225.3173253

27. David Ledo, Fraser Anderson, Ryan Schmidt, Lora Oehlberg, Saul Greenberg, and Tovi Grossman. 2017. Pineal: Bringing Passive Objects to Life with Embedded Mobile Devices. In Proceedings of the 2017 CHI Conference on Human Factors in Computing Systems. ACM, 2583-2593. DOI :

http://dx.doi.org/10.1145/3025453.3025652

28. David Lindlbauer, Jörg Müller, and Marc Alexa. 2016. Changing the appearance of physical interfaces through controlled transparency. Proceedings of the 29th Annual Symposium on User Interface Software and Technology (2016), 425-435. DOI : http://dx.doi.org/10.1145/2984511.2984556

29. Joseph Luk. 2004. Computer-controlled airbrush with motion tracking. Dept. Computer Science, Univ. British Columbia (2004).

30. LumiLor. 2019. Electroluminescent paint: https://www.lumilor.com/. Last accessed September 2019. (2019).

31. MG Chemicals. 2019. Thermochromic - 3D Printer Filaments : www.mgchemicals.com/products/3d-printingsupplies/3d-printer-filaments/thermochromic. Last accessed September 2019. (2019).

32. Christos Mourouzi, Isabel PS Qamar, and Anne Roudaut. 2018. SweepScreen: Sweeping Programmable Surfaces to Create Low-fi Displays Everywhere. Extended Abstracts of the 2018 CHI Conference on Human Factors in Computing Systems (2018), LBW603. D0I:

http://dx.doi.org/10.1145/3170427.3188462

33. Simon Olberding, Sergio Soto Ortega, Klaus Hildebrandt, and Jürgen Steimle. 2015. Foldio: Digital fabrication of interactive and shape-changing objects with foldable printed electronics. In Proceedings of the 28th Annual ACM Symposium on User Interface Software \& Technology. ACM, 223-232. DOI :

http://dx.doi.org/10.1145/2807442 .2807494

34. Simon Olberding, Michael Wessely, and Jürgen Steimle. 2014. PrintScreen: fabricating highly customizable thin-film touch-displays. Proceedings of the 27th annual ACM symposium on User interface software and technology (2014), 281-290. DOI:

http://dx.doi.org/10.1145/2642918.2647413

35. Jianyong Ouyang, T-F Guo, Yang Yang, Hiroyuki Higuchi, Masahiro Yoshioka, and Tatsuki Nagatsuka. 2002. High-performance, flexible polymer light-emitting diodes fabricated by a continuous polymer coating process. Advanced Materials 14, 12 (2002), 915-918. DOI : http://dx.doi .org/10.1002/1521-4095(20020618)14: 12<915: : AID-ADMA915>3.0.C0;2-9

36. Roshan Lalintha Peiris and Ryohei Nakatsu. 2013. TempTouch: a novel touch sensor using temperature controllers for surface based textile displays. Proceedings of the 2013 ACM international conference on Interactive tabletops and surfaces (2013), 105-114. DOI :

http://dx.doi.org/10.1145/2512349.2512813

37. Romain Prévost, Alec Jacobson, Wojciech Jarosz, and Olga Sorkine-Hornung. 2016. Large-scale painting of photographs by interactive optimization. Computers \& Graphics 55 (2016), 108-117. DOI :

http://dx.doi.org/10.1016/j.cag.2015.11.001

38. ProtoPasta. 2019. : https://www.proto-pasta.com/pages/conductive-pla. Last accessed September 2019. (2019).

39. Parinya Punpongsanon, Xin Wen, David S Kim, and Stefanie Mueller. 2018. ColorMod: Recoloring 3D Printed Objects using Photochromic Inks. Proceedings of the 2018 CHI Conference on Human Factors in Computing Systems (2018), 213. DOI : http://dx.doi.org/10.1145/3173574.3173787

40. Anne Roudaut, Abhijit Karnik, Markus Löchtefeld, and Sriram Subramanian. 2013. Morphees: toward high shape resolution in self-actuated flexible mobile devices. Proceedings of the SIGCHI Conference on Human Factors in Computing Systems (2013), 593-602. DOI : http://dx.doi.org/10.1145/2470654.2470738

41. Andreas Sandström, Amir Asadpoordarvish, Jenny Enevold, and Ludvig Edman. 2014. Spraying Light: Ambient-Air Fabrication of Large-Area Emissive Devices on Complex-Shaped Surfaces. Advanced Materials 26, 29 (2014), 4975-4980. DOI :

http://dx.doi.org/10.1002/adma. 201401286 
42. Valkyrie Savage, Ryan Schmidt, Tovi Grossman, George Fitzmaurice, and Björn Hartmann. 2014. A series of tubes: adding interactivity to 3D prints using internal pipes. In Proceedings of the 27th annual ACM symposium on User interface software and technology. ACM, 3-12. DOI: http://dx.doi.org/10.1145/2642918.2647374

43. Martin Schmitz, Mohammadreza Khalilbeigi, Matthias Balwierz, Roman Lissermann, Max Mühlhäuser, and Jürgen Steimle. 2015. Capricate: A fabrication pipeline to design and 3D print capacitive touch sensors for interactive objects. Proceedings of the 28th Annual ACM Symposium on User Interface Software \& Technology (2015), 253-258. DOI : http://dx.doi.org/10.1145/2807442.2807503

44. Ethan B Secor. 2018. Principles of aerosol jet printing. Flexible and Printed Electronics 3, 3 (2018), 035002. DOI : http://dx.doi .org/10.1088/2058-8585/aace28

45. Roy Shilkrot, Pattie Maes, Joseph A Paradiso, and Amit Zoran. 2015. Augmented airbrush for computer aided painting (CAP). ACM Transactions on Graphics (TOG) 34, 2 (2015), 19. DOI : http://dx.doi .org/10.1145/2699649

46. Yuta Sugiura, Koki Toda, Takayuki Hoshi, Youichi Kamiyama, Takeo Igarashi, and Masahiko Inami. 2014. Graffiti fur: turning your carpet into a computer display. In Proceedings of the 27th annual ACM symposium on User interface software and technology. ACM, 149-156. DOI : http://dx.doi.org/10.1145/2642918.2647370

47. David Sweeney, Nicholas Chen, Steve Hodges, and Tobias Grosse-Puppendahl. 2016. Displays as a material: A route to making displays more pervasive. IEEE Pervasive Computing 15, 3 (2016), 77-82. DOI: http://dx.doi.org/https://doi.org/10.1109/MPRV.2016.56

48. Voxel8:. 2019. https://www.voxel8.com/. Last accessed September 2019. (2019).

49. Guanyun Wang, Lining Yao, Wen Wang, Jifei Ou, Chin-Yi Cheng, and Hiroshi Ishii. 2016. xPrint: A modularized liquid printer for smart materials deposition. Proceedings of the 2016 CHI Conference on Human Factors in Computing Systems (2016), 5743-5752. DOI : http://dx.doi.org/10.1145/2858036.2858281

50. Martin Weigel, Tong Lu, Gilles Bailly, Antti Oulasvirta, Carmel Majidi, and Jürgen Steimle. 2015. iSkin: flexible, stretchable and visually customizable on-body touch sensors for mobile computing. In Proceedings of the 33rd Annual ACM Conference on Human Factors in Computing Systems. ACM, 2991-3000. DOI : http://dx.doi.org/10.1145/2702123.2702391

51. Martin Weigel, Aditya Shekhar Nittala, Alex Olwal, and Jürgen Steimle. 2017. Skinmarks: Enabling interactions on body landmarks using conformal skin electronics. In Proceedings of the 2017 CHI Conference on Human Factors in Computing Systems. ACM, 3095-3105. DOI : http://dx.doi.org/10.1145/3025453.3025704

52. Michael Wessely, Theophanis Tsandilas, and Wendy E Mackay. 2016. Stretchis: Fabricating highly stretchable user interfaces. (2016), 697-704. DOI :

http://dx.doi.org/10.1145/2984511.2984521

53. Karl Willis, Eric Brockmeyer, Scott Hudson, and Ivan Poupyrev. 2012. Printed optics: 3D printing of embedded optical elements for interactive devices. Proceedings of the 25th annual ACM symposium on User interface software and technology (2012), 589-598. DOI: http://dx.doi.org/10.1145/2380116.2380190

54. Oktay Yarimaga, Maesoon Im, Yang-Kyu Choi, Tae Won Kim, Yun Kyung Jung, Hyun Gyu Park, Sumi Lee, and Jong-Man Kim. 2010. A color display system based on thermochromic conjugated polydiacetylene supramolecules. Macromolecular research 18, 4 (2010), 404-407. DOI :

http://dx.doi.org/10.1007/s13233-010-0402-7

55. Yizhong Zhang, Chunji Yin, Changxi Zheng, and Kun Zhou. 2015. Computational hydrographic printing. ACM Transactions on Graphics (TOG) 34, 4 (2015), 131. DOI : http://dx.doi.org/10.1145/2766932 